Orthopäde $2010 \cdot 39: 549-550$

DOI 10.1007/s00132-009-1584-6

Online publiziert: 8. Mai 2010

(c) Springer-Verlag 2010

\author{
M. Rauschmann · J. Richolt \\ Abteilung für Wirbelsäulenorthopädie, Orthopädische Universitätsklinik \\ Friedrichsheim gGmbH, Frankfurt/Main
}

\title{
Dorsale dynamische Systeme
}

Die operative Therapie der degenerativen Lendenwirbelsäule und Ihrer Begleiterscheinungen ist sehr facettenreich und geprägt von vielen Philosophien und Ideen, welche bis zum heutigen Tage noch keine klaren Aussagen zum wirklichen Nutzen auf lange Sicht zulassen. Die Lücke zwischen der rein dekomprimierenden und der stabilisierenden Operation haben sog. dorsale dynamische Systeme eingenommen. Diese Gruppe der dynamischen Systeme lassen sich prinzipiell in interspinöse Implantate, pedikelschraubenbasierte Systeme, den Facettengelenkersatz und Hybridsysteme unterteilen. Alle biomechanischen Studien zu diesen Implantatsystemen zeigen prinzipiell einen Benefit in Bezug auf die Restabilisation eines erkrankten Bewegungssegments bzw. beweisen eine indirekte Erweiterung des Spinalkanals, der Neuroforamina bzw. eine Reorientierung der teleskopartig ineinander geschobenen Facettengelenke. Je nach Untergruppe dienen diese Implantate als ,add on“ einer Dekompression um eine Restenose zu verhindern bzw. den häufig postoperativ auftretenden lumbalgieformen Schmerz zu reduzieren.

Die pedikelschraubenbasierten Implantatsysteme sollen einerseits die degenerierten Gelenke entlasten und diese in ihrer Bewegung führen und auch in dem Bereich der „neutralen Zone“ begrenzen. Des Weiteren wird einigen Systemen eine Entlastung der Bandscheibe und somit eine Möglichkeit der Regeneration nachgesagt. Die dritte Gruppe versucht die Funktion der Facettengelenke durch Ihren Ersatz zu kompensieren und somit die natürliche Beweglichkeit unter der Verhinderung einer Instabilität zu erhalten. Eine weitere Gruppe stellt die Kombination aus Fusion und dynamischer Stabilisation dar. Diese sog. Hybridsysteme oder im Falle von interspinösen Implantaten bezeichneten „Topping-off-Lösungen“ zielen v. a. auf die Verhinderung einer Anschlussinstabilität, sowie auf die Verkürzung von Spondylodesestrecken ab.

Ein Problem existiert aus heutiger Sicht nicht nur in den fehlenden Langzeitergebnissen, die aus prospektiven Studien gewonnen werden müssen, sondern auch in der klaren Differentialindikation zu den oben genannten Optionen. Hier fehlen bis heute klare Vorgaben, welches System wann am besten zum Einsatz kommen soll. Die Schwierigkeit liegt alleine schon in der klaren Klassifikation des Grades der Degeneration der Bandscheibe und der Facettengelenke oder der häufig hieraus resultierenden Instabilität. Alle genannten Aspekte lassen viel Raum für die Interpretation, so dass die Indikationsstellung sehr subjektiv gefärbt ist und nicht auf harten Fakten beruht. Klar erscheinen indes einige Kontraindikationen wie beispielsweise das degenerative Wirbelgleiten höheren Grades oder die degenerative Lumbalskoliose.

\section{$>$ Update zur reinen Dekompressionsoperation}

Dieses Themenheft adressiert genau diese Problematik. Es soll einerseits ein Update zur reinen Dekompressionsoperation liefern, die weiterhin als der Goldstandard der Therapie der Spinalkanalstenose anzusehen ist, gefolgt von biomechanischen Aspekten zu den am häufigsten eingesetzten interspinösen Platzhaltern. Vier dieser interspinösen Platzhalter (X-STOP, Wallis, DIAM und Coflex) wurden ausge- 
wählt und im einzelnen präsentiert. Die Arbeiten stellen Gemeinsamkeiten aber auch Differentialindikationen des jeweiligen Implantates heraus und zeigen die zum jetzigen Zeitpunkt vorliegenden klinischen Ergebnisse aus teils eigenen, teils publizierten Daten.

Die dynamischen Stabsysteme sind exemplarisch durch das „Dynesys-System" vertreten. Auch hier werden Philosophie, Langzeitergebnisse, soweit vorhanden und eigene Erfahrungen zur Darstellung gebracht.

Der Innovation des Facettengelenkersatzes, der am meisten von allen Technologien im experimentellen Stadium ist, wird durch einen Übersichtsbeitrag Rechnung getragen. Schlussendlich wird der Sachstand zur Hybridversorgung bzw. dem „topping off“ präsentiert und diskutiert.

Alle genannten Arbeiten geben einen guten Überblick zum derzeitigen Stand der Erkenntnis und setzen sich kritisch mit dieser neuen Technologie auseinander. Hierfür danken die Schriftleiter dieses Themenheftes den beteiligten Autoren für Ihre offene und klare Berichterstattung unter Einbeziehung eigener Ergebnisse und Erfahrungen.

Viele Aspekte konnten trotz der teils umfangreichen Beiträge nicht erschöpfend behandelt werden. Auch die Darstellung der Implantate hat keinen Anspruch auf Vollständigkeit, sondern soll diejenigen zur Darstellung bringen, welche sich auf dem Wirbelsäulenmarkt schon etabliert haben.

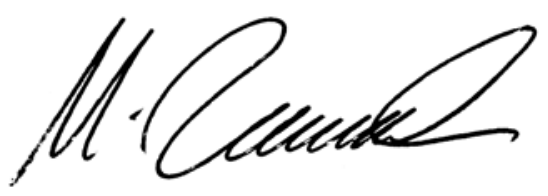

M. Rauschmann

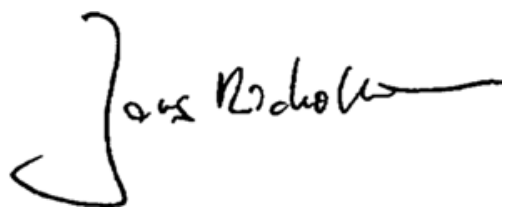

J. Richolt

\section{Korrespondenzadresse}

\section{PD Dr. M. Rauschmann}

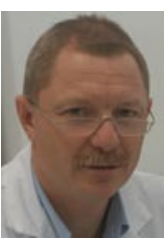

Abteilung für Wirbelsäulenorthopädie, Orthopädische Universitätsklinik Friedrichsheim gGmbH

Marienburgstraße 2, 60528 Frankfurt/Main m.rauschmann@friedrichsheim.de

Interessenkonflik. Der korrespondierende Autor weist auf folgende Beziehung hin: Der Autor ist Vortragender bei Fachsymposien der Firma Paradigma Spine zur Thematik dorsaler dynamischer Systeme in der Vergangenheit gewesen.

\section{Ethik in der Intensivmedizin}

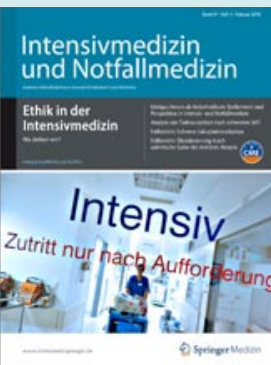

Die mittlere Lebenserwartung hat in den Industrienationen während der letzten Jahre deutlich zugenommen, was zu einem erhöhten Anteil älterer $\mathrm{Pa}$ -

tienten in den Kliniken führt. Durch neue Errungenschaften in der Intensivmedizin werden die natürlichen Grenzen des Lebens stetig weiter verschoben. Dadurch steigt bei vielen Menschen jedoch auch die Angst vor einer inhumanen Apparatemedizin, die den Sterbeprozess verlängert und unnötiges Leiden schafft. Die behandelnden Ärzte auf der Intensivstation müssen deshalb neben medizinischem Wissen vermehrt auch mit juristischen Kenntnissen und sozialer Kompetenz aufwarten, um Patienten und Angehörigen bei Ihren Entscheidungen kompetent zur Seite stehen zu können.

Die Zeitschrift Intensivmedizin und Notfallmedizin informiert mit dem Leitthemenheft "Ethik in der Intensivmedizin" (Heft 1/2010) sowohl theoretisch als auch praxisorientiert über die Komplexität des Sterbens auf der Intensivstation.

Die Themen des Heftes sind u. A.:

- Ethische Bewertung von Entscheidungen am Lebensende von Intensivpatienten

- Palliativmedizin

- Angehörigenkonferenz

- Organisation medizinischer Entscheidungen am Lebensende

Bestellen Sie diese Ausgabe zum Preis von $30,-€$ bei

Springer Customer Service Center Kundenservice Zeitschriften

Haberstraße 7

69126 Heidelberg

Tel.: +49 6221-345-4303

Fax: +49 6221-345-4229

E-Mail: leserservice@springer.com

www.Intensivmedizin.Springer.de 